\title{
Tardiness heuristic for scheduling Flexible Manufacturing Systems
}

\author{
ALI S. KIRAN, SEMA ALPTEKIN and A. CELAI. KAPLAN
}

\begin{abstract}
This paper evaluates the tardiness performance of a sampling-based adaptive heuristic in a dynamic manufacturing environment. A test bed, following a real world manufacturing system, has been developed. The proposed algorithm has been implemented in this simulated cnvironment. After fine tuning the algorithm, it has been tested in various shop conditions. The results of these simulation studies are summarized.
\end{abstract}

\section{Introduction}

The effective utilization of manufacturing systems requires efficient scheduling and control systems. Such systems must be capable of responding to changes in a complex dynamic manufacturing environment. These 'changes' include machine breakdowns, unavailability of material and other resources to perform manufacturing operations, changes in demand, customer priorities and required delivery dates. To be able to respond to these changes effectively, a scheduling system must be able to develop new feasible schedules in a short time. Furthermore, the proposed schedule should provide a reasonably good performance of the system for the selected criteria. Our experience with the feedback heuristic (Kiran and Alptekin, 1989) indicates that the feedback heuristic is a viable alternative for the scheduling of flexible manufacturing systems.
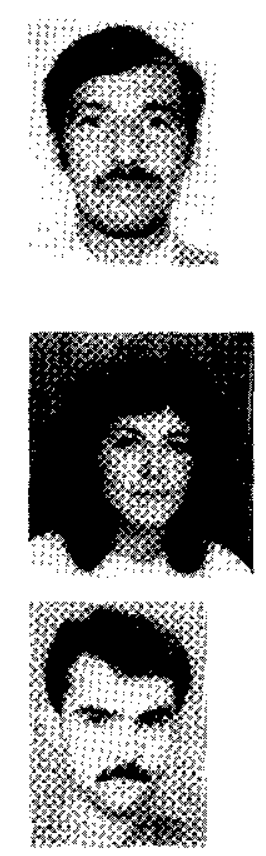

Authors: Ali S. Kiran, Kiran and Associates, San Diego, USA, Sema Alptekin, Department of Engineering Management, University of Missouri-Rolla, Rolla, MO 65401, USA, A. Celal Kaplan, Department of Industrial and Systems Engineering, University of Southern California, Los Angeles, CA 90089-0193, USA.

Ali S. KIRAN is Professor and Chairman of the Industrial Engineering Department at Dai-Yeh Institute of Technology, and is President of Kiran \& Associates, representing ProModelPC and other CIM integration tools. Prior to his current appointments, Dr Kiran held academic appointments at the University of Southern California, Texas Technical University, St. Mary's University of San Antonio and Istanbul Technical University. He received his MS degree in Mechanical Engineering and his $\mathrm{PhD}$ in Industrial Fnginecring from Istanbul Technical University. Dr Kiran's research and consulting activities include production planning and control, and scheduling and simulation of manufacturing systems. He is a frequent speaker at international conferences in industrial engineering, manufacturing systems, operations research and simulation.

SEMA Al_ptEKIN is an Associate Professor of Engineering Management and academic director of the computer integrated manufacturing laboratory at the University of Missouri-Rolla. She holds a BS and a MS in Mechanical finginecring and a PhD in Industrial Engineering from Istanbul Technical University. Her current research interests are in the area of computer integrated manufacturing, intelligent manufacturing systems and production scheduling. She was the recipient of the 1988 Outstanding Young Manufacturing Engineer Award given by the Society of Manufacturing Engineers. She is a member of IIE, ORSA, SME and ASEE.

A. Celal KAPt AN is a PhD Candidate in the Industrial and Systems Engineering department of the University of Southern California. His rescarch interests are in the areas of scheduling for manufacturing systems and simulation. He holds a BS and MS in Industrial Engineering from the Middle East Technical University. He is a member of IIE and ORSA. 


\section{Background}

Flexible manufacturing systems (FMS) are developed to take advantage of flexible automation. In contrast to fixed automation, FMSs are characterized by almost non-existent changeover times from one part type to another. Hence, batch sizes can be recluced without losing the economical advantages of fixed automation. Smaller batch sizes and the simultaneous production of different part types will in turn result in shorter production lead times, improvement of due date performance and increased customer/user satisfaction. In addition, other benefits of fixed automation such as part interchangcability and quality are still valid in flexible automation.

The issues that must be addressed during the phase of an FMS life cycle are given in Table 1 as a hierarchical decision structure (Kiran et al., 1988). Assuming that design and aggregate planning decisions have already becn made, the next task is to solve setup and scheduling problems sequentially with proper coordination maintained between them. The sequential treatment of setup and scheduling problems is necessitated by the computational properties of these problems. Our approach is based on the above mentioned hierarchical structure, and is intended to solve FMS scheduling problems and to integrate the resulting scheduling decisions with the system setup decisions.

System setup refers to a segment of the master schedulc for which certain resource allocation decisions have to be made (Kiran and Tansel, 1986). T'hese decisions are

1. Part type selection: detcrmining a set of parts to produce in the system setup period.

2. Tooling: assignment required tools to machines.

3. Fixture allocation: allocation of the limited number of fixtures to part types.

Table 1. Hierarchical decision structure.

\begin{tabular}{ll}
\hline Hierarchical level & lecisions \\
\hline Design & $\begin{array}{l}\text { System contiguration: machine types, } \\
\text { layout, MHS and storage system } \\
\text { design } \\
\text { Factory-wide production plans, batch } \\
\text { sizes, due dates, capacities } \\
\text { Parts to be produced during short- } \\
\text { term planning horizon, operation tool } \\
\text { assignment } \\
\text { Job priorities, start and completion } \\
\text { times of operations } \\
\text { Real-time control of activities in the } \\
\text { system }\end{array}$ \\
\hline
\end{tabular}

4. Operations assignment: assigning operations to machines which have been equipped with the proper tools.

5. Routing: determining part routings in the system.

The solution of the system setup problems will yield

- the set of parts which will be produced during the setup period;

- a machine routing for each part;

- an allocation of tools to machines which will achicve the production goals set by the master schedule;

- an allocation of fixtures to parts:

- an assignment of operations to machines.

After solving the setup problems, the next task is to determine start and completion tirnes for each activity. We refer to this stage as the 'scheduling stage.' 'The solution of the scheduling problem is input to the FMS controller, which controls the real time operations of the system.

In the scheduling context, manufacturing systems can be modelled as generalized job shops. In a job shop, a set of jobs (parts, products, etc.) may require several different operations which are performed by a set of machines (processors, workstations, etc.). Numerous studics on the scheduling of job shops are reported. We refer to Lawler et al. (1982), Graves (1981), Kiran and Smith (1984) and Blazewicz et al., (1988) for surveys of scheduling problems.

A schedule determines the start/completion times for each operation of each job waiting to be processed in the shop. An optimal schedule minimizes (maximizes) a function of job completion times. A classification of criteria commonly used in job shop scheduling is given in Table 2. Panwalkar et al. (1973) reported that the due date based criteria is considered the most important by the practitioners, followed by the minimization of setup

Table 2, Job shop scheduling criteria.

I. Criteria based on job performance

I.A. Criteriat based on jon completion times e.g. average waiting-itme

I.B. Criteria based on Work-In-Progress e.g. average number of jobs in the shop

I.C. Criteria based on shop-performance e.g. machine utilization

1I. Criteria based on due dates

e.g. mean tardiness

III. Cost based criteria e.g. total setup cost 
times and the minimization of in-process inventories, in that order. Similar findings have more recently been reported by Smith et al. (1983).

Table 3 provides the notation and basic definitions which will be used throughout this paper. We consider job tardiness-based criteria in this paper. This is due to the practical importance of tardiness related performance measures in real world manufacturing settings. A set of the most commonly used tardiness based criteria is given in Table 4. As can be seen, the mean tardincss per tardy job and root mean square of tardiness penalizes schedules with a few jobs that are very late. Maximum tardiness, although simplistic, has been used extensively in assembly driven shops. Average normalized tardiness allows for the comparison of different shops with different job processing times.

The shop scheduling problem is notorious for its complexity; i.e. time complexity of an exact solution algorithm is bounded by $O\left(n !^{M}\right)$. This led to the development of heuristic job shop dispatching rules which could not guarantec an optimal schedule. To determine a schedule, a dispatching rule assigns a priority value to each waiting job in each machine queuc. When a

Table 3. Notation and basic definitions.

\begin{tabular}{ll}
$n$ & the number of jobs \\
$m$ & the number of machines \\
$P_{i j}$ & processing time of operation $j$ of job $i$ \\
$m_{i}$ & the number of operations of job $i$ \\
$d_{i}$ & due date of job $i$ \\
$c_{i j}$ & completion time of operation $j$ of job $i$ \\
$c_{i}$ & completion time of last operation of job $i$ \\
$r_{i}$ & arrival time (ready time) of job $i$ \\
$F_{i}=c_{i}-r_{i}$ & job flow time \\
$L_{i}=c_{i}-d_{i}$ & job lateness \\
$T_{i}=\max \left\{0, L_{i}\right\}$ & job tardiness \\
\hline
\end{tabular}

'Table 4. Most commonly used tardiness-based criteria.

\begin{tabular}{ll}
\hline Mean tardiness & $\bar{T}=\sum_{i=1}^{n} T_{i} / n$ \\
& $\% T=n_{T} / n$ \\
Percent tardy & $n_{T}=\sum_{i=1}^{n} 1_{i}\left(T_{i}\right)$ \\
The number of tardy jobs & $\bar{T} / n_{T}$ \\
Mean tardiness per tardy & $\left(\sum_{i=1}^{n} T_{i}^{2} / n\right)^{1 / 2}$ \\
Root mean square of tardiness & $T_{\max }=\max \left\{T_{i}\right\}$ \\
Maximum tardiness & $\bar{T}($ Normalized $)=\frac{T}{1} \sum_{i=1}^{n} \sum_{j=1}^{m_{i}} P_{i j}$ \\
\end{tabular}

machine bccomes available, the job with the highest priority is scheduled. Dispatching rules can be easily applied in real-time cnvironments, hence they are widely used in practice. Panwalkar and Iskander (1977) classified and summarized 113 dispatching rules, most of which were devcloped and tested on simulated job shops. Some of the most widely referred to dispatching rules are summarized in Table 5. FCFS is a benchmark rule with a performance no different than a randomly generated feasible schedule. The SPT rule is known to be efficient with respect to tardiness related criteria in highly congested shops (Kiran and Smith 1984). SPT-T has been designed to avoid long waiting times for a few very long jobs (Oral and Malouin, 1973). EDD and SLACK are commonly used in practice. Baker (1984) and Baker and Bertrand (1982) reported success with the MODD and an extended version of the MODD. The estimated tardiness cost in COVERT (Carroll, 1965) is a measure of the cstimated job tardiness rather than an actual cost. Estimated job waiting times are also utilized in ATC (Vepsalainen and Morton, 1988).

The FMS scheduling problem differs from conventional problems in that there are additional resources and more complicated task processing in FMSs (Blazewicz et al., 1988). The FMS scheduling problem may be approached from two different views

1. The scheduling of all operations of available jobs at the beginning of a predetermined scheduling period, i.e. a priori scheduling.

2. I'o schedule operations, one at a time, when they become available, i.e. on-line dispatching.

Mirchandani (1989) classified the scheduling issues of FMSs, and Morton and Smunt (1986) described a four level hicrarchical decision structure in which both a priori and on-line scheduling have been recognized.

The first approach, a priori scheduling, requires perfect information on all available parts, machine tools, fixtures, pallets and the material handling system's status. A priori schedule is vulnerable to system disturbances resulting from breakdowns or unpredicted demand changes, and has to be adjusted. But there is no guarantee that the adjusted schedule will be optimal. Furthermore, finding the optimal schedule is not easy; it has been shown that FMS scheduling problems are at least as hard as job shop scheduling problems. This is because of the additional resource constraints in the FMS scheduling problem, such as fixture and pallet availability; limits on the automated material handling system and in-process storage space avallabilitics. In addition, flexible part routings and alternative machines further increase the number of alternative feasible schedules and computational requirements of the solution algorithms. 
Table 5. Priority dispatching rules used in this study.

\begin{tabular}{|c|c|c|c|}
\hline Rule & & Rank & Priority of operation $k$ of job $i$ at time $t$ \\
\hline L,WR & Least Working Remaining & $\min$ & $\sum_{j=k}^{m_{i}} p_{i j}$ \\
\hline SPT & Shortest Processing Time & $\min$ & $p_{i k}$ \\
\hline SI.ACK & Job Slack & $\min$ & $d_{i}-\sum_{j=k}^{m_{i}} p_{i j}-l$ \\
\hline EIDD & Earlicst Due Date & $\min$ & $d_{i}$ \\
\hline CR & Critical Ratio & $\min$ & $\left(d_{i} \cdots t\right) / \sum_{j=k}^{n_{i}} p_{i j}$ \\
\hline COVERT & Cost OVER lime & $\max$ & $c_{i} / p_{i j}$ \\
\hline S/OPN & Slark/Operation & min & $\left(d_{i} \cdots \sum_{j=k}^{m_{i}} p_{i j}-t\right) /\left(m_{i}-k+1\right)$ \\
\hline MODD & Modified Operation Due Date & $\min$ & $\begin{cases}d_{i k} & \text { If } S L A C K_{i}>0 \\
d_{i k}+p_{i k} & \text { otherwise }\end{cases}$ \\
\hline \multirow[t]{2}{*}{ A'TC } & Apparent Tardiness Cost & $\max$ & $\frac{v_{i}}{p_{i j}} \exp -\frac{\left(d_{i}-t-p_{i j}-\sum_{j=k+1}^{m_{i}}\left(W_{i j}+p_{i j}\right)\right)}{a p}$ \\
\hline & wherc & $\begin{array}{l}p_{i k} \\
m_{i} \\
c_{i} \\
\mathrm{a} \\
\mathrm{p} \\
v_{i} \\
W_{i j}\end{array}$ & $\begin{array}{l}\text { processing time of operation } k \text { of job } i \\
: \text { the number of operations required to complete job } i \\
\text { : expected tardiness cost of job } i \\
\text { : a coefficient } \\
\text { : average prorcssing time } \\
: \text { weight of job } i \\
\text { : expected waiting time of opcration } j \text { of job } i\end{array}$ \\
\hline
\end{tabular}

The mixed integer program developed by Chang et al. (1984) is a good example of the complexity of the problem. For a five workstation-10 part type FMS scheduling problem, the model has 30000 binary decision variables, 500 continuous decision variables, and 25000 constraints.

Mathematical programming and analytical models have been employed to study a priori scheduling problems. Afentakis (1986), Chang et al. (1984) and Raman et al. (1986) considered the relations between the system sctup phase and the scheduling phase in their integer programming formulations. Frschler et al. (1984) anat lysed the periodic release of parts into the system. Tang and Denardo $(1988 a, b)$ developed job scheduling models for a single machine FMS problem to minimize the number of tool switches and the number of switching instants. Kusiak (1986, 1989) defined a combined scheduling problem in a flexible machining/assembly system.

The actual state of the system can casily be taken into consideration using the on-line approach. But the drawback here is the myopic nature of the decision making process. The schedule resulting from the on-line dispatching of operations may not bc efficient. Even more seriously, on-line dispatching may cause system deadlocks or the 'starvation' of the system (if additional necessary precautionary measures have not been taken). On-line dispatching rules have received somewhat more attention than a priori scheduling in FMS research. This is partly because of the availability of simulation techniques (Grant 1988), the body of knowledge on dispatching rules in job shops, and the applicability of dispatching rules in AI-based approaches. AI-based approaches have becn proposed by Kusiak and Chen (1988), Shaw (1986), Shaw and Whinston (1986), Shen and Chang (1986) and Subramanyam and Askin (1986). Merabet (1986) proposed changing dispatching rules depcnding on the shop status. Shaw (1988) developed a search algorithm for static problems. A distributed approach for FMS scheduling is proposed by Shaw (1987). Simple dispatching rules have been developed and tcsted by Denzler et al. (1987), Hutchinson and Wyne (1973), Lin and Lu (1984), Raman et al. (1986), Stecke (1981) and Wang (1986).

The problem with the dispatching rules is that none of them are superior to others for all schcduling critcria. 
Even for a single criterion, it is not uncommon to find conflicting results in the literature. The relative performance of a heuristic also depends on experimental conditions. Each of the dispatching rules of Table 5 has a strong bias to generate the same kind of schedules which may not be successful under different shop conditions. Kiran and Alptekin (1986) proposed a sampling-based heuristic to overcome this problem. Thcir Feedback Heuristic $(\mathrm{FH})$ is different in that a small subset of feasible schedules is generated using an adaptive priority function.

\section{The feedback heuristic}

The objective of this paper is to develop a scheduling tcchnique that is

1. easy to understand and apply;

2. adaptable to changing problem parameters;

3. performs well under different shop conditions.

We propose a sampling-based heuristic as a viable alternative for such a scheduling technique; the Feedback Heuristic is based on generating a subset of schedules and selecting the best among them. We choose to generate non-delay schedules, i.e. schedules in which no machine is kept idle if there is an available job. By sampling through the non-delay schedules we hope to reach a schedule with an acceptable performance.

In a sense, the $\mathrm{FH}$ is similar to other sampling-based techniques. It differs from the others in that the generation of a new schedule in the $\mathrm{FH}$ is based on a cumulative evaluation of earlier schedules. This feedback mechanism is the most likely cause of the superior performance of this feedback heuristic. The feedback heuristic is summarized below

\section{FEEDBACK HEURISTIC FOR TARDINESS CRITERION:}

Let $I_{t}$ be the set of parts (jobs) which are to be scheduled at time $t$.

STEP 0 (initialization): find an active schedule for the available jobs. Set $P_{i}$ iteration index, $k=1$. Calculate $T_{i}, k$ and $P_{i}, k$ for each job and average tardiness.

STEP 1: Calculate job priority for each job using (1)

$$
P_{i k}=(1-\alpha) P_{i, k-1}+\alpha\left(T_{i, k-1}\right)^{\beta}
$$

where

$$
P_{i 0}=0 \text { for all } i
$$

$P_{i k}$ : is the priority for iteration $k$

$T_{i, k-1}$ : the tardiness of job $i$ at iteration $k-1$

$\alpha$ and $\beta$ are two non-negative coefficients.
STEP 2 (scheduling): List the available jobs in a nonincreasing order of job priority values. Schedule the jobs in this list.

STEP 3: Calculate the tardiness for cach job and the average tardiness for the new schedule. Compare the average tardiness for the new and the previous schedules. Record the new schedule if its average tardiness is smaller than the previous one, then go to Step 1.

STEP 4: If $k<\Gamma, k=k+1$, go to STEP 1. Otherwise, STOP and display the last recorded schedule.

The implementation of this algorithm in a dynamic manufacturing environment is not straightforward and requires the simultaneous scheduling of all required resources. These resources are

1. Tools: in our hierarchical structure, tools are assigned to machines during the system setup phase simultaneously with the operations: if a machine is assigned to an operation, the required tools must bc available to that machine during the scheduling horizon. Hence, there is no need to consider tools explicitly during the scheduling phase.

2. Fixture-pallets: the system setup model assigns parts to fixture types. Time allocation decisions for fixtures must be considercd during the scheduling phase since each pallet-fixture may serve more than one part during the scheduling horizon.

3. Material handling vehicles: these need to be scheduled because each job requires at least two transports between the load/unload stations and machining centres. The difficulty in scheduling material handling vehicles is that the transport time is a function of the operation start and completion times on machining centres, which in turn are dependent on the availability of the material handling vehicles.

4. Storage (input-output buffer) spaces: in a FMS, each station has at least one, rrostly two buffer spaces. The scheduling of the buffer spaces is requircd to avoid system deadlocks. Buffer spaces cannot be considered as a machine in a classical scheduling problem due to the fact that there is no operation that is required on them. In other words, the buffer spaces can be considered as machines with variable processing times between 0 and $\infty$.

5. Stations: these are the elements with assigned machining, load/unload, inspection, etc. operations. For each part, a fixed operation time can be determined on the stations. They are considered as 'machines' in a classical scheduling problem.

In this study, the $\mathrm{FH}$ has been implemented as follows: develop schedules for machines then modify them for 
feasibility with respect to tools, fixture-pallets, material handling vehicles, storage spaces and stations. We refer the interested reader to Kiran and Alptekin (1986) for a detailed discussion on the implementation of this algorithm.

\section{Computational results}

We tested the FH in a multi-stage experimental framework

1. Preliminary evaluation of the FH on static problems against a set of well known priority rules.

2. Pilot evaluation of the FH against a selected subset of the priority rules in 1 and against iterativeCOVERT on a simulated model of a real world FMS.

3. Further evaluation of the FH against COVERT' in a simulated dynamic FMS enviromment.

Initially we compared the $\mathrm{FH}$ with other single-pass priority rules in static problcms. These tests were conducted for two reasons

1. To verify the superior performance of the $\mathrm{FH}$ to any other single pass heuristic.

2. To fine tune the $\mathrm{FH}$ and to test the effect of:

a. Number of iterations

b. Starting schedule

c. different values of $\alpha$ and $\beta$.

More extensive tests of the FH werc conducted on a simulated model of a real world FMS. On these more realistic test conditions we evaluated the performance of the $\mathrm{FH}$ against a subset of the single pass heuristics, as well as against iterative-COVERT algorithm.

We chose COVERT due to its superior performance in the earlier studies (Carroll, 1965). The iterative implementation here follows Morton (1988)

1. Use COVERT with a lead time estimate to develop an initial schedule.

2. Update estimated lead times, repeat Step 1 for equal number of steps that are used in the $\mathrm{FH}$.

\subsection{Preliminary evaluation and fine tuning of the $\mathrm{FH}$ on the static problems}

Static problems were generated considering the typical FMS scheduling problems (Smith et al., 1986) with the number of jobs ranging from 6 ro 10 . The number of machines were chosen between three and five. Two buffer spaces for each machine are considered. The material handling system has two automated guided vehicles.

6, 8 and 10-job and 3,4 and 5-machine problems with simultaneous arrivals have been considered. A total of 1089 problems, 121 problems in each subclass, have been tested. We have generated 10 job types with a random number of operations, job routings, processing times and due dates for 3, 4 and 5-machine problems. The job type set held constant for a given number of machines through this set of cxperiments. Processing times and due dates werc generated using uniform distributions (in the dynamic experiments, exponentially distributed processing times and different due date determination rules have also been tested). The feedback heuristic was compared with five other simple dispatching rules given in Table 5. The EDD rule was used in the initialization step of the feedback heuristic and the number of iterations were set to 30 for this set of problems.

To make a fair evaluation of the FIH we also generated 30 random schedules. The best of these schedules was chosen and compared to the schedule given by the $\mathrm{FH}$. We used the paired-t test for this comparison. The paired-t test has also been applied to the difference between the tardiness value of the $\mathrm{FH}$ and the tardiness of the best schedule that was found by all of the dispatching rules.

The average total tardiness values for each problem size are shown in Table 6 , where the average total tardiness of the feedback heuristic is consistently better than the best of the other heuristics and random schedules. Table 7 shows the number of problems for which the best solution has been found by each heuristic. As can be seen from Tables 6 and 7 , the feedback heuristic performed increasingly better with the increasing number of jobs and machines.

As stated earlicr, we also considered the following fine tuning aspects of the algorithm

1. the number of itcrations, $\Gamma$

2. the feedback coefficient, $\alpha$

3. the tardiness coefficient, $\beta$

4. the initial schedule.

It was expectcd that the performance of the algorithm would improve by increasing the number of iterations. However, we found that $\Gamma=30$ provides a reasonably good schedule and increasing $\Gamma$ over 150 does not change the performance of the algorithm significantly (confidence level $=0.95$ ). We concluded that the number of iterations can be chosen between 30 and 150 depending on the accuracy requirements and the problem size. 
Table 6. Average total tardiness performance of the heuristics on static problems.

\begin{tabular}{|c|c|c|c|c|c|c|c|c|}
\hline $\begin{array}{l}\text { \# of } \\
\text { machines }\end{array}$ & $\begin{array}{l}\text { \# of } \\
\text { jobs }\end{array}$ & SPT & LWR & SLACK & $\mathrm{S} / \mathrm{OPN}$ & $\mathrm{CR}$ & RND & $\mathrm{FH}$ \\
\hline & 5 & 9.31 & 11.49 & 8.49 & 8.96 & 8.93 & 11.17 & 6.81 \\
\hline \multirow[t]{3}{*}{3} & 8 & 38.15 & 44.19 & 40.56 & 41.50 & 41.25 & 46.39 & 32.73 \\
\hline & 10 & 74.93 & 83.33 & 84.06 & 85.50 & 83.83 & 88.26 & 67.43 \\
\hline & 5 & 5.99 & 6.46 & 4.03 & 3.96 & 4.67 & 6.68 & 2.59 \\
\hline \multirow[t]{3}{*}{4} & 8 & 27.72 & 32.07 & 28.36 & 26.06 & 26.69 & 35.03 & 18.47 \\
\hline & 10 & 59.45 & 65.18 & 67.95 & 61.63 & 60.89 & 70.68 & 44.84 \\
\hline & 5 & 10.88 & 12.94 & 11.08 & 10.89 & 10.81 & 13.82 & 8.74 \\
\hline \multirow[t]{2}{*}{5} & 8 & 50.10 & 53.85 & 58.20 & 54.70 & 54.95 & 57.05 & 42.26 \\
\hline & 10 & 91.93 & 94.78 & 112.58 & 103.96 & 102.17 & 106.47 & 79.26 \\
\hline
\end{tabular}

Table 7. Comparison of the heuristics on static problems.

\begin{tabular}{|c|c|c|c|c|c|c|c|c|}
\hline $\begin{array}{l}\text { \# of } \\
\text { machines }\end{array}$ & $\begin{array}{l}\text { \# of } \\
\text { jobs }\end{array}$ & SPT & LWR & SLACK & $\mathrm{S} / \mathrm{OPN}$ & CR & RND & $\mathrm{FH}$ \\
\hline & 5 & 48 & 20 & 73 & 69 & 68 & 29 & 116 \\
\hline \multirow[t]{3}{*}{3} & 8 & 15 & 3 & 15 & 17 & 17 & 2 & 104 \\
\hline & 10 & 24 & 3 & 4 & 6 & 6 & 2 & 94 \\
\hline & 5 & 51 & 52 & 84 & 79 & 75 & 37 & 121 \\
\hline \multirow[t]{3}{*}{4} & 8 & 6 & 7 & 16 & 19 & 15 & 2 & 118 \\
\hline & 10 & 5 & 0 & 2 & 3 & 1 & 2 & 113 \\
\hline & 5 & 62 & 36 & 70 & 66 & 61 & 30 & 115 \\
\hline \multirow[t]{2}{*}{5} & 8 & 28 & 15 & 15 & 9 & 11 & 10 & 63 \\
\hline & 10 & 9 & 11 & 2 & 5 & 4 & 1 & 105 \\
\hline
\end{tabular}

We tested the effect of different sets of $\alpha$ and $\beta$ values on the performance of the FH. Initially we tested $\alpha$ values of $0.01,0.05,0.1,0.2,0.25,0.4,0.5$ and 0.8 . The performance of the $\mathrm{FH}$ deteriorated for $\alpha=0.01$ and $\alpha>0.25$. In the case of $\alpha=0.01$ the same schedule may be generated several times because of the small rate of change in priority values. $\alpha>0.25$ causes oscillating priority values, hence the $\mathrm{FH}$ cycles through only a handful of different schedules. In the range $0.05-0.25$ there was not any statistically significant (confidence level $=0.95$ ) performance difference. This may be due to the nature of the smoothing process which was used in calculating the priorities. The priorities are given by

$$
P_{i k}=(1-\alpha) P_{i, k-1}+\alpha\left(T_{i, k-1}\right)^{\beta}
$$

Rewriting the priority function, we have

$$
P_{i, k+1}=(1-\alpha)^{2} P_{i, k-1}+(1-\alpha) \alpha T_{i, k-1}^{\beta}+\alpha T_{i, k}^{\beta \beta}
$$

and

$$
P_{i, \Gamma}=\alpha \sum_{k=1}^{\Gamma}(1-\alpha)^{\mathbf{\Gamma}-k} T_{i, k-1}^{\beta}
$$

Hence the rankings of the priority values of a set of jobs may remain the same over the range of $0.05-0.25$. We choose $\alpha=0.1$ for the rest of the study.

A similar analysis for $\beta$ indicated that the values 0.5 ,
1 and 2 are not significantly different, hence we chose $\beta=1$ because of its computational advantages.

The initial schedule significantly changes the performance of the FH for a small total number of iterations. For $\boldsymbol{\Gamma}=150$, however, we could not detect any significant performance difference between the different initial schedules.

\subsection{Simulation of a dynamic FMS}

We compared the performance of the FH on a detailed simulation model of an existing FMS. Fig. 1 shows a schematic representation of the simulated system.

The simulated system consists of five machining centres and two load/unload stations. The material handling operations are performed by two automated guided vehicles (AGV). Similar to the existing system, we assumed that every morning a daily list of new jobs is given to the scheduler. 'The jobs are randomly chosen from a pool of 10 different part types. These parts require 2-6 machining operations. We considered both constant (deterministic) and exponentially distributed processing times. Each machine has one input and one output buffer. 'l'here is also a central buffer which can hold up 


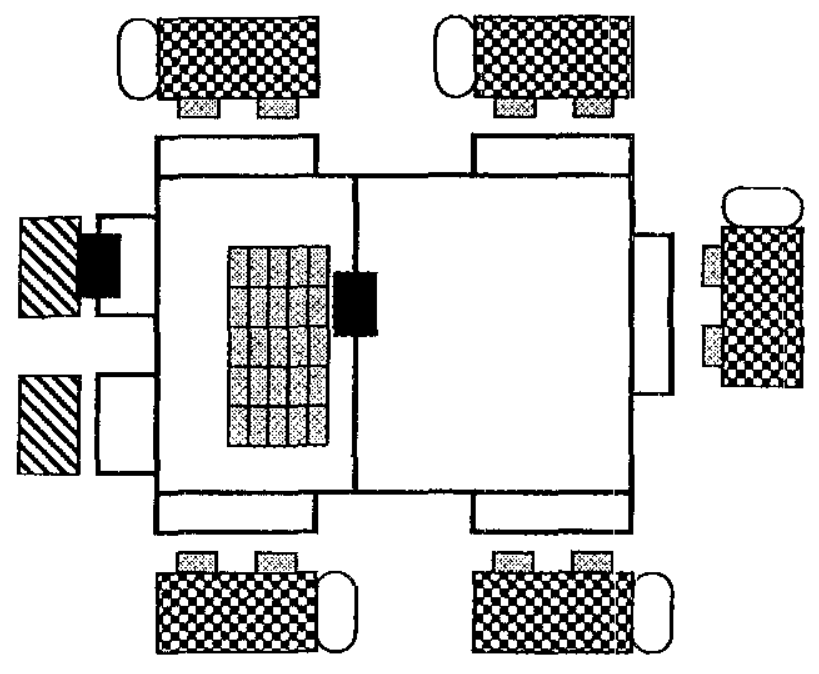

D LOAD/UNLOAD STATION

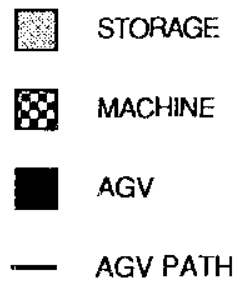

Figure 1. Schematic representation of the simulated system.

to 20 parts. The number of parts in the system is limited to 25 .

Observation of the real system revealed that a uniform workload assumption cannot be justified. Hence in some simulated scenarios we assumed that the average workload of the machines are different. The load factors are set to $1.05,1,0.975,0.85$ and 0.825 for machines 1 to 5 , respectively. We also simulated the scenarios with balanced workloads to generalize our findings. These simulation experiments were conducted for a $85 \%$ utilization of the bottleneck machine(s).

Due date for each new job was determined by a function of its total work content, i.e.

$$
d_{i}=\text { current time }+(\text { lead time })_{i}
$$

where

$$
\begin{aligned}
(\text { lead time })_{i}= & \left((\text { total processing time })_{i}\right. \\
& + \text { Random }(0-100) \\
& *(\text { number of jobs in the shop } / 18)
\end{aligned}
$$

This function was based on observations made of the existing system.
Pilot experiments

First we ran a set of pilot experiments to eliminate non-promising priority rules. In these set of experiments, the simulation time was set to 16 weeks. The first four weeks were used for warming up the system. Statistics were collected during the next 10 weeks. The last two weeks were used to avoid a clean-up effect. We assumed that the system operated five days per week and 16 hours per day.

We ran two different scenarios for deterministic and exponential processing time assumptions. The $\mathrm{FH}$ was configured with $\alpha=0.1, \beta=1, \Gamma=150$ and the EDD initial schedule.

Figs 2, 3 and 4 show results for the selected priority rules for the deterministic operation times. The results are similar for the other scenario with the exponential processing times and not given here for the purpose of brevity.

\section{The FHI versus COVERT}

The results of the pilot experiments indicate that the $\mathrm{FH}$ and the COVERT are far superior to other simple dispatching rulcs. Hence, we further evaluated these two rules for a variety of tardiness based criteria given in Table 4, namely the mean tardiness, the percent tardy, the mean tardiness per tardy, and the average normalized tardiness. We also included the FCFS and the SPT as benchmarks. In these experiments, balanced shop configurations were added to the experimental design. Hence a full factorial design of four scenarios was simulated. Table 8 shows the experimental design.

For each scenario 11 replications were made. The number of replications were chosen by observing the ratio (Law and Kelton, 1982; pp 288-290)

$$
\frac{\text { confidence interval half-length }}{\text { Average }}
$$

for performance measures that we used. For 11 replications, the above ratio was around 0.15 , which indicated a low variation.

Each replication consisted of a simulated time of 20 days of warm-up period, followed by 150 days of steady state operations and 10 days of clean-up time. The warm up period was found adequate by observing individual tardiness statistics of the jobs. All other experimental conditions were similar to those of the pilot experiments. Statistics were collected during the 150 day steady state operations period. The feedback heuristic is configured with $\alpha=0.1, \beta=1$ and $\Gamma=80$. The COVERT rulc was initially configured with a work content based waiting time estimation rulc given by 
Expected Waiting Time = remaining processing time $e_{i}$

$$
\times\left(\frac{\text { number of jobs in the shop }}{15}\right)
$$

'lables $9,10,11$ and 12 show the results of these experiments for the mean tardiness per tardy, the percent tardy, the mean tardiness and the average normalized tardiness (see Tablc 4 for the definitions). In the following table, the results for the FCFS and the SPT are also included as benchmarks.

As can be seen in Table 9, the FH dominates the COVERT for the mean tardiness per tardy criterion.

Fig. 5 shows the $90 \%$ confidence intervals for this criterion. The non-overlapping confidence intervals in Fig. 5 indicate that 'the $\mathrm{FH}$ performs better than the COVERT' in all of the four scenarios.

Table 10 indicates that for the percent tardy criterion, the COVERT performs significantly better than the FH in all scenarios. This, however, is expected duc to the implementation of the iterative priority function used in the $\mathrm{FH}$. By increasing the $\beta$ value, more emphasis could be given to late jobs in the sampling process, hence some tardy jobs could be avoided in exchange for the larger tardiness values per tardy job.

Results for the mean tardiness (Table 11) and the average normalized tardiness ('lable 12) are somewhat similar to those given in Table 9. For the mean tardiness, the $\mathrm{FH}$ performs significantly ( $90 \%$ confidence) better than the COVERT $T$ in scenarios 1, 3 and 4 . For scenario 2, although the COVERT shows a better average behavior than the $\mathrm{FH}$, we cannot reject the null hypothesis that 'there is no significant difference between the FH and the COVERT' at the $90 \%$ confidence level. The average normalized tardiness results also confirms the dominance of the $\mathrm{FH}$ in the same three scenarios.

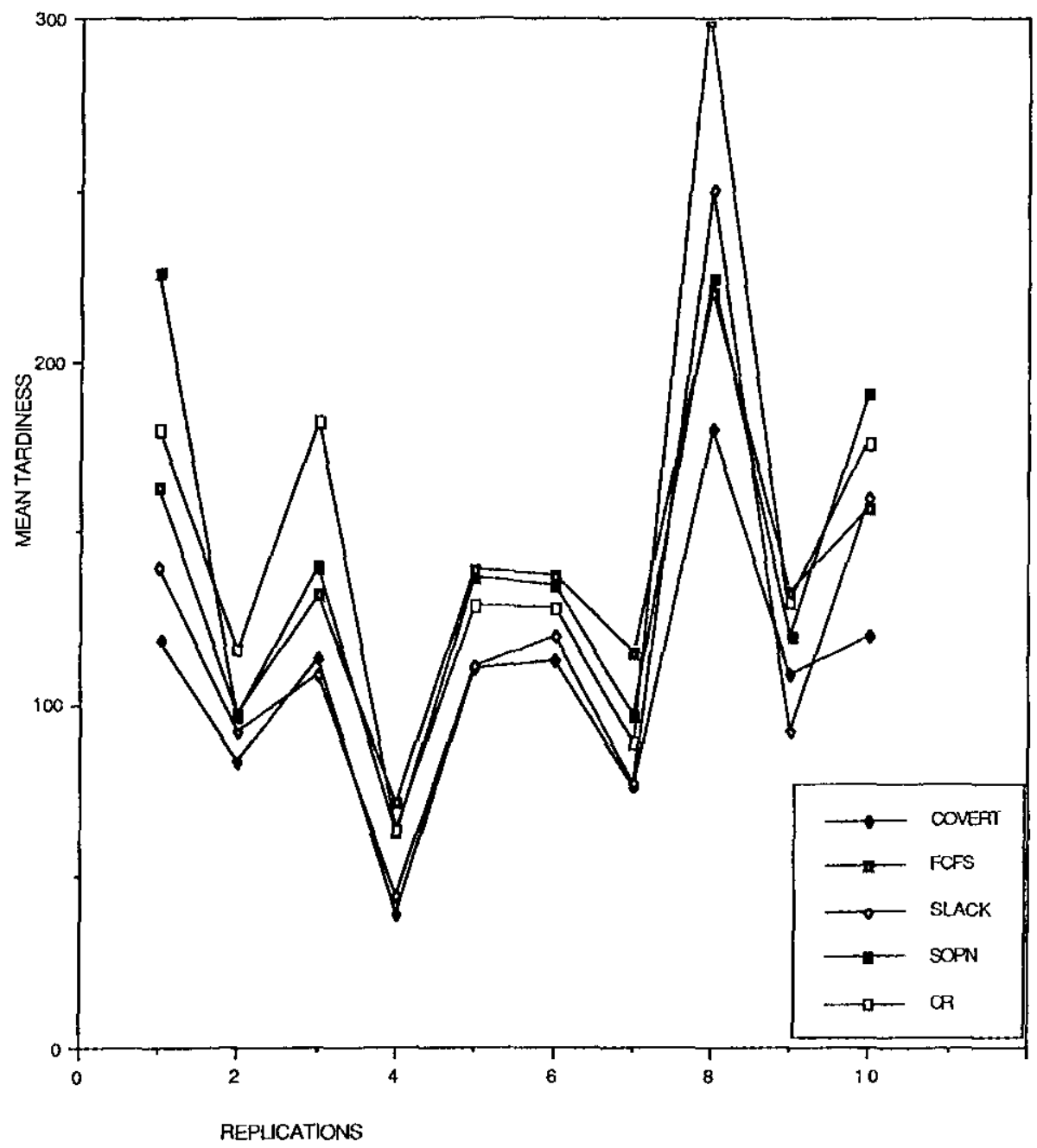

Figure 2. Results for mean tardiness per week: the COVERT versus the sclected priority rules. 
In general, the experiments indicate that the FH performs well for the tardiness-related criteria for which it was designed. Relatively small variance of the performance measures indicate a consistent behavior of the FH.

\section{Summary and future research}

We have developed a feedback-based heuristic for scheduling flexible manufacturing systems. 'The heuristic has been tested on randomly generated problems as well as on a simulated model of an existing system. The algorithm has been found to be very effective in obtaining consistently better solutions than other rules tested in this study. We have implemented the algorithm in a real world like environment to test the feasibility of applying the algorithm in practice. The experimentation indicates that the feedback heuristic performs reasonably well for a variety of tardiness based criteria. Furthermore, the computational burden of the algorithm is not prohibitive, even for large problems.

Full scale implementation of the algorithm requires more work, however. We are currently working on similar algorithms for other scheduling performance measures such as job throughput times and cost measures. Different schedule generating schemes are also under investigation. The idea of using the performance of the current schedulc to define the relative priorities of the jobs in the next schedule seems to be worth further investigation.

We believe that the efficiency of algorithms will be improved using information gathered from past experience. This can be achieved by defining an adaptive feed-

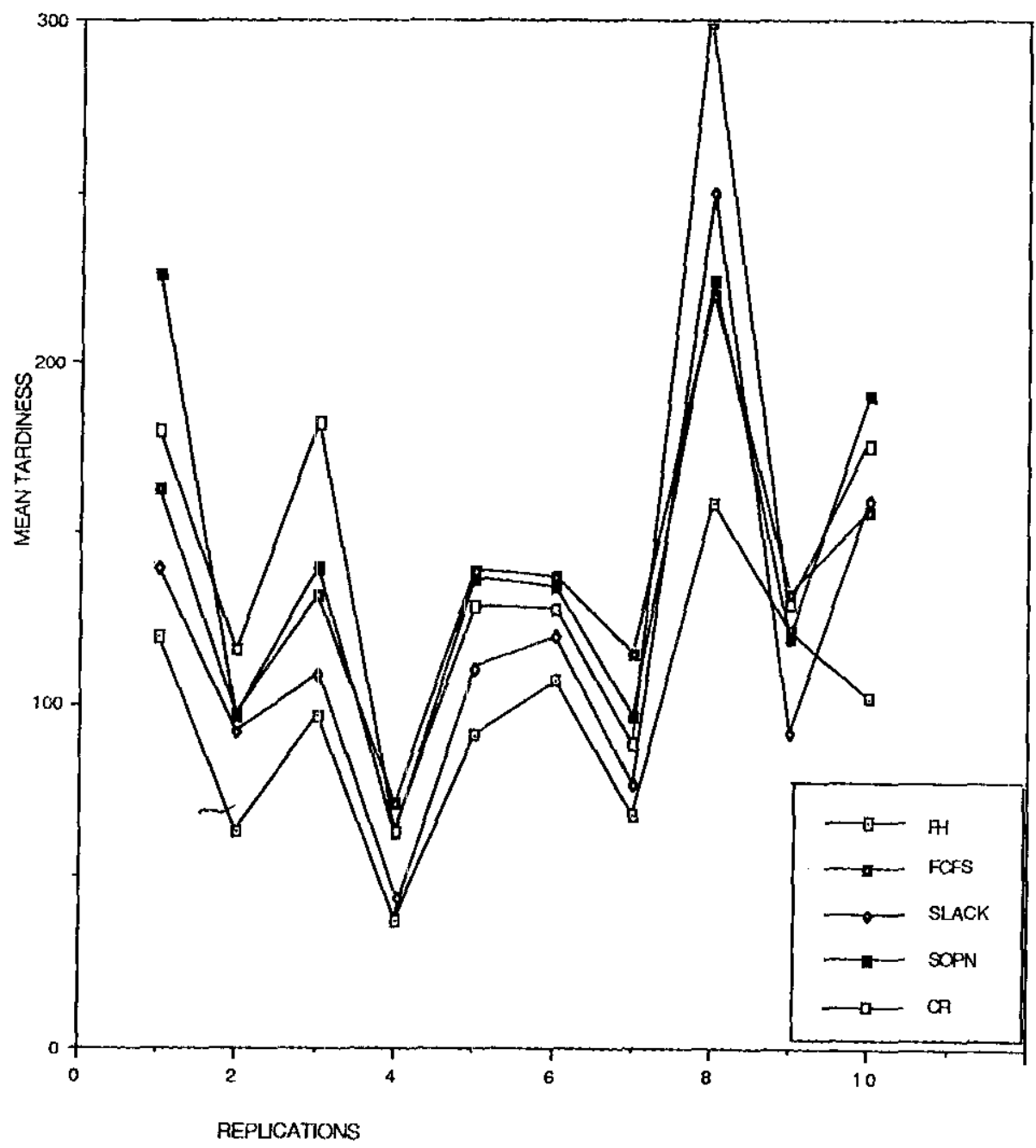

Figure 3. Results for mean tardiness per week: the $\mathrm{FH}$ versus the selceted priority rules. 
back mechanism in the generation of active schedules. A feedback coefficient may be defined as a function of the FMS state variables at time $t$. These are the subject of future studies in the application of artificial intelligence to FMS scheduling problems.
T'able 8. Experimental design.

\begin{tabular}{lll} 
& Shop & \multicolumn{1}{c}{ Processing times } \\
\hline Scenario 1 & balanced & deterministic \\
Scenario 2 & balanced & exponential \\
Scenario 3 & unbalanced & deterministic \\
Scenario 4 & unbalanced & exponential \\
\hline
\end{tabular}

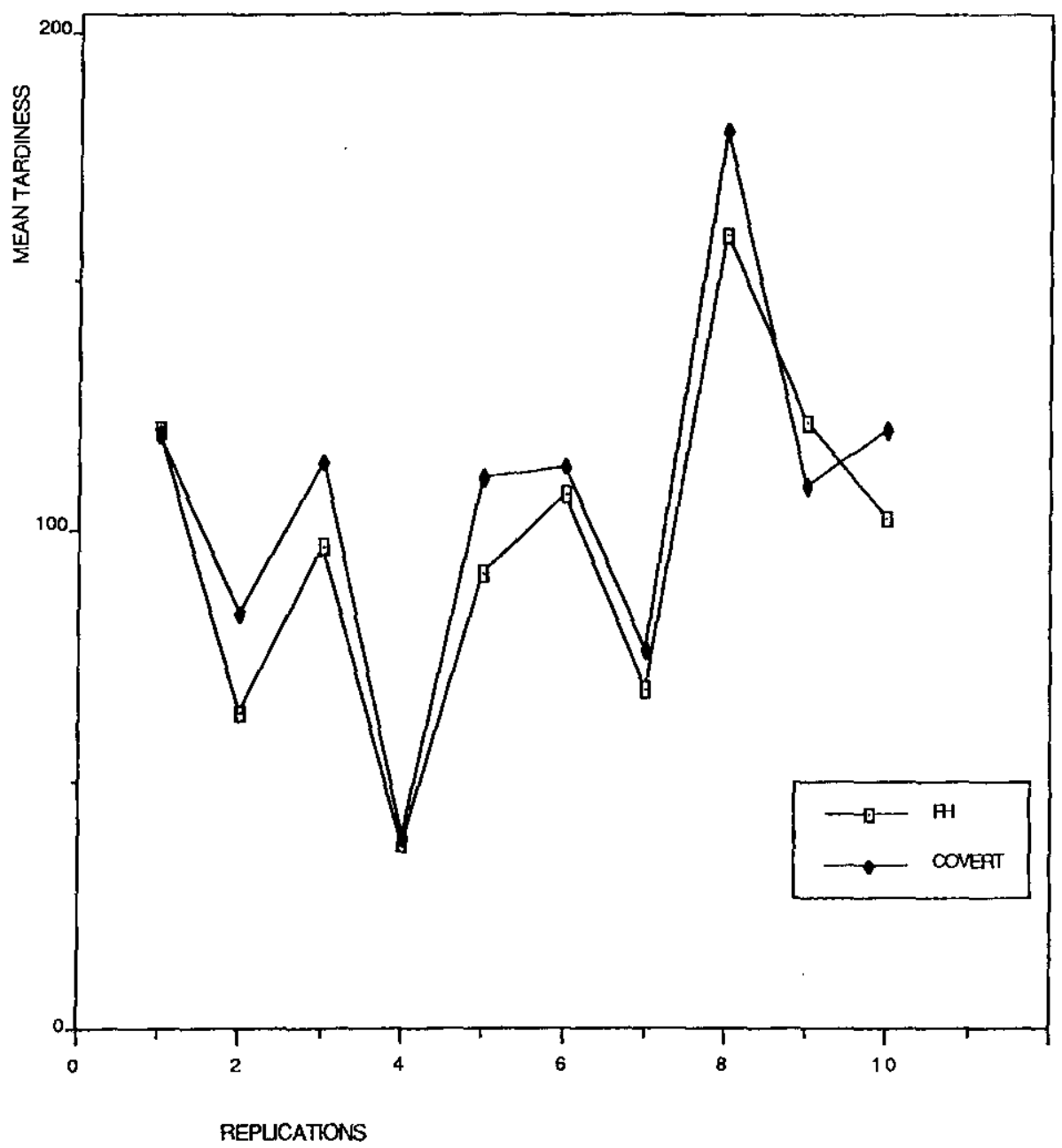

Figure 4. Results for mean tardiness per week: the COVER' versus the FH.

Table 9. The mean tardiness per tardy results: mean (standard deviation).

\begin{tabular}{|c|c|c|c|c|c|c|c|c|}
\hline \multirow{2}{*}{$\frac{\text { Scenario }}{1}$} & \multicolumn{2}{|c|}{ FCFS } & \multicolumn{2}{|c|}{ SPT } & \multicolumn{2}{|c|}{ COVERT } & \multicolumn{2}{|c|}{ FHI } \\
\hline & 431.78 & $(31.57)$ & 882.85 & $(194.50)$ & 368.81 & $(21.89)$ & 156.73 & $(17.90)$ \\
\hline 2 & 813.90 & $(121.59)$ & 1824.59 & $(400.66)$ & 632.76 & $(100.70)$ & 409.63 & $(21.14)$ \\
\hline 3 & 281.83 & $(21.92)$ & 345.69 & $(31.55)$ & 227.58 & $(22.44)$ & 63.44 & $(4.95)$ \\
\hline 4 & 461.19 & $(62.69)$ & 624.12 & $(93.37)$ & 324.31 & $(27.86)$ & 184.44 & $(28.37)$ \\
\hline
\end{tabular}


Table 10. The percent tardy results: mean (standard deviation).

\begin{tabular}{lllllllll}
\hline Scenario & \multicolumn{2}{c}{ FCFS } & \multicolumn{2}{c}{ SPT } & \multicolumn{2}{c}{ COVERT } & \multicolumn{2}{c}{ FH } \\
\hline 1 & 43.42 & $(1.17)$ & 35.79 & $(0.99)$ & 27.64 & $(1.45)$ & 40.81 & $(1.61)$ \\
2 & 52.78 & $(1.77)$ & 36.66 & $(1.34)$ & 49.16 & $(3.84)$ & 56.21 & $(1.55)$ \\
3 & 42.44 & $(0.76)$ & 35.30 & $(1.07)$ & 24.91 & $(0.60)$ & 36.29 & $(1.68)$ \\
4 & 47.24 & $(1.39)$ & 39.80 & $(0.78)$ & 42.16 & $(1.85)$ & 52.00 & $(3.44)$ \\
\hline
\end{tabular}

Table 11. The mean tardiness results: mean (standard deviation).

\begin{tabular}{|c|c|c|c|c|c|c|c|c|}
\hline \multirow{2}{*}{ Scenario } & \multicolumn{2}{|c|}{ FCFS } & \multicolumn{2}{|c|}{ SP' I } & \multicolumn{2}{|c|}{ COVERT } & \multicolumn{2}{|c|}{$\mathrm{FH}$} \\
\hline & 211.89 & $(9.08)$ & 323.61 & $(61.02)$ & 119.71 & $(10.93)$ & 75.51 & (10.44) \\
\hline 2 & 489.98 & $(80.12)$ & 673.10 & (144.11) & 360.30 & $(83.77)$ & 376.60 & $(29.11)$ \\
\hline 3 & 154.65 & $(12.21)$ & 156.40 & $(11.59)$ & 69.02 & (6. 46$)$ & 33.37 & $(4.82)$ \\
\hline 4 & 263.08 & $(49.13)$ & 294.12 & $(35.51)$ & 164.27 & $(18.10)$ & 121.32 & $(25.60)$ \\
\hline
\end{tabular}

Table 12. The average normalized tardiness results: mean (standard deviation).

\begin{tabular}{|c|c|c|c|c|c|c|c|c|}
\hline \multirow{2}{*}{$\frac{\text { Scenario }}{1}$} & \multicolumn{2}{|c|}{ FCFS } & \multicolumn{2}{|c|}{$\mathrm{SPT}$} & \multicolumn{2}{|c|}{ COVERT } & \multicolumn{2}{|c|}{$\mathrm{FH}$} \\
\hline & 139.06 & $(5.21)$ & 221.12 & $(40.83)$ & 82.20 & $(7.19)$ & 54.135 & $(6.63)$ \\
\hline 2 & 335.87 & $(55.46)$ & 463.97 & $(83.73)$ & 247.07 & $(58.13)$ & 238.30 & $(17.37)$ \\
\hline 3 & 129.57 & $(9.74)$ & 132.26 & (9.33) & 64.40 & $(4.98)$ & 33.70 & $(3.31)$ \\
\hline 4 & 224.57 & $(40.66)$ & 250.62 & $(31.44)$ & 143.16 & $(13.99)$ & 110.38 & $(20.98)$ \\
\hline
\end{tabular}

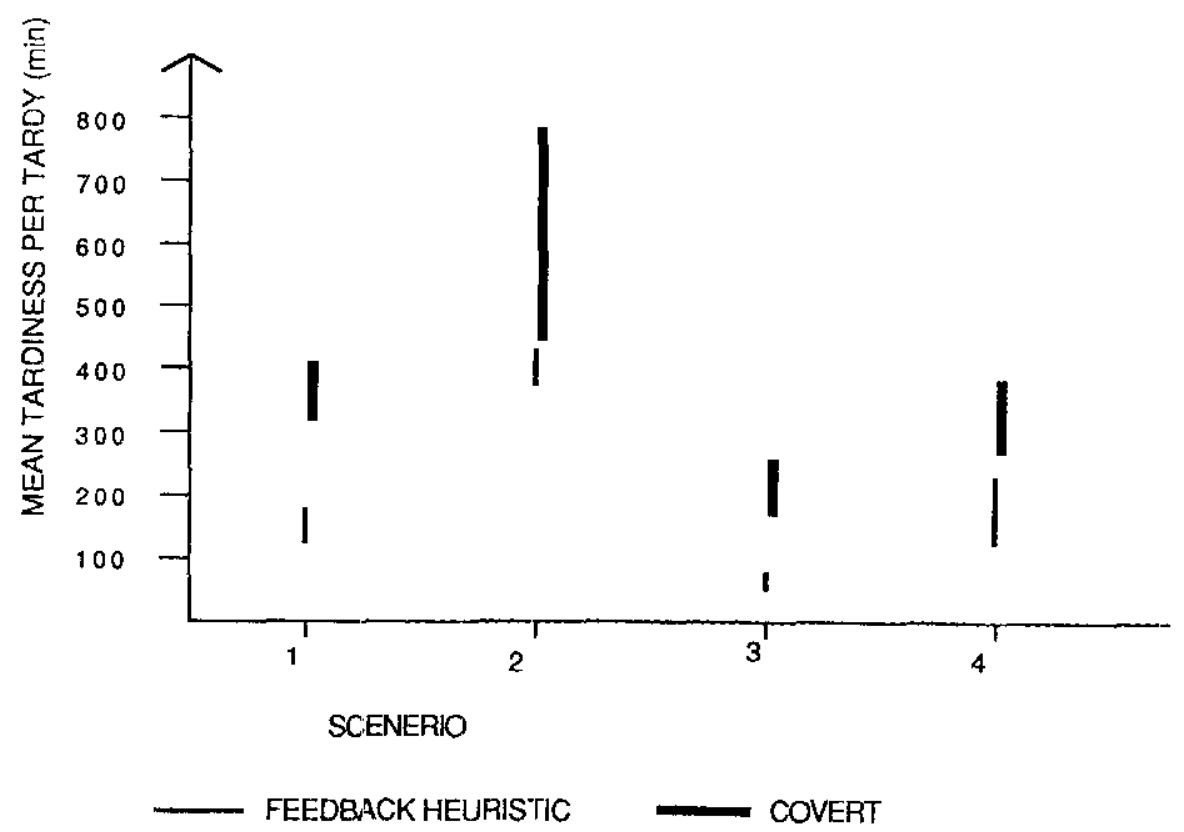

Figure 5. $90 \%$ confidence intervals for $\mathrm{FH}$ and COVERT'. 


\section{Acknowledgment}

The authors are indebted to Selcuk Karabati for his help in coding and running computer programs. This work was partially supported by the National Science Foundation under Grant DMC-87-09171.

\section{References}

Afritakis, P., 1986, Maximum throughput in flexible manufacturing systems, Proceedings Sccond ORSA/TIMS Conference on Flexible Manufacturing Systems, K. E. Stecke and R. Suri (eds), 509-520 (Amsterdam: Elsevier).

Ammons, J. C., Govindraj, T., and Mitchell, C. M., 1986, Human aided scheduling for FMS: a paradigm for human-computer interaction in real time scheduling and control, Proceedings Second ORSA/TIMS Conference on Flexible Manufacturing Systems, K. E. Stecke and R. Suri (eds), 443-454 (Amsterdam: Elsevier).

BAKER, K. R., 1984, Sequencing rules and due-date assignments in a job-shop, Management Science, 30, 9, 1093-1104.

BAKER, K. R., and BERTRAND, J. W. M., 1982, A dynamic priority rule for scheduling against due-dates, Journal of Oper. ations Management, 3, 1, 37-42.

Bi.AzEWIC7, J., Finke, G., Haupt, R., and SCImidt, G., 1988, New trends in machine scheduling, European Journal of Operations Research, 37, 303-317.

Chang, Y. I., Sullivan, R. S., and Bagcil, U., 1984, Experimental investigation of quasi-realtime scheduling in FMS, Proceedings First ORSA/TIMS Conference on FMS, August.

CARROLL, D. C., 1965, Heuristic sequencing of single and multiple component jobs, Ph.D. Thesis, MIT, USA, June.

DenZILER, D. R., and BOE, W. J., 1987, Experimental investigation of FMS scheduling rules, International Journal of Production Research, 25, 7, 979-994.

GRAVES, S. C., 1981, A review of production scheduling, Operations Research, 29, 4, 646-675.

ERsChler, J,, Roubellat, F., and Thurio'i, C., 1984 , Steady state of a flexible manufacturing system with periodic releasing and flow time constraints, Proceedings First ORSA/TIMS Conference on FMS, 327-345, August.

French, S., 1982, Sequencing and Scheduling, (Chichester: Ellis Horwood).

Grant, F. H., 1988, Simulation in designing and scheduling manufacturing systems, in W. D. Compton (ed.), Design and Analysis of Integrated Manufacturing Systems (National Acaderny Press). .

Hutchinson, G. K., and Wynne, B. F., 1973, A flexible manufacturing system, Industrial Engineering, 10-17, December.

KIRAN, A. S., and Alp'tekin, S., 1986, Scheduling jobs in flexiblc manufacturing systems, NBS Special Publication No. 724, 383-400, September.

KIRAN, A. S., and ALPTEKiN, S., 1989, A tardiness heuristic for scheduling flexible manufacturing systerns, 15th NSF Grantees Conference on Production Research and Technology, Bcrkeley, CA, January.

KIRAN, A. S., and Smirh, M. L., 1984, Simulation studies in job shop scheduling, Comp. and Ind. End. 8, 2, 87-93.

Kiran, A. S., Schloffer, A., and Hawkins, D., 1988, An integrated simulation approach to design, planning and scheduling of flexible manufacturing systems, Working Paper 88-02, Department of Industrial and Systems Engineering, University of Southern California.

KIRAN, A. S., and TANSEI, B. C., 1986, The system setup in FMS: concepts and formulation, Proceedings Second ORSA/TIMS Conference on Flexible Manufacturing Systems, K. E. Stecke and R. Suri (eds), 321-332 (Amsterdam: Elsevier).

KUSIAK, A., 1986, Scheduling flexible machining and assembly systems, Proceedings Second ORSA/TIMS Conference on Flexible Manufacturing Systems, K. F. Stccke and R. Suri (eds), $521-532$ (Amsterdam: Elscvier).

KusIak, A., 1989, Aggregate scheduling of a flexible machining and assembly system, IEEE Transactions on Robotics and Automation, 5, 4, 451 .459.

Kustak, A., and CHE.v, M., 1988, KBSS: A knowledge-based system for manufacturing scheduling, Working Paper \#05/88, University of Manitoba.

LAW, M. A., and KF.TON, W. D., 1982, Simulation Modeling and Analysis, (New York: McGraw-Hill).

I.AWLER, E. L., LFNDTRA, J. K., and RinNOOY KaN, A. H. G., 1982, Recent developments in deterministic sequencing and scheduling: a survey, in M. A. H. Dempster, J. K. I.enstra and A. H. G. Rinnooy Kan (eds), Deterministic and Stochastic Siheduling, 35-74 (Dordecht: Reidel).

LiN, I. S., and LU, C. Y., 1984, The scheduling problem in random flexible manufacturing systerns, Proceedings First ORSA/TIMS Conference on FMS, August.

Mrkabet, A. A., 1986, Dynamic job shop scheduling: an operating system based design, in A. Kusiak (ed.), Flexible Manufacturing Systems: Methods and Studies, 257-270 (Amsterdam: North-Holland).

Mirchandani, P. B., 1989, Concurrent tooling and scheduling of flexible machines, CORS/TIMS/ORSA Joint National Meeting, Vancouver, Canada, May 8-10.

Morton, T. E., and SMun'r, T. L., 1986, in A. Kusiak (ed.), Flexible Manufacturing Systems: Methods and Studies, 151-164 (Amsterdam: North-Holland).

MORTON, T. E., 1988, Filtered beam search in scheduling, International Journal of Production Research, 26 (1), 35-62.

ORAL, M., and Malolin, J. L., 1973, Evaluation of the shortest processing time scheduling rule with truncation process, AIEE Trans., 5, 4, 357-365.

PanWal.Kak, S. S., and Iskandfr, W., 1977, A survey of scheduling rules, Operations Research, 25, 1, 45-61.

Panwalkar, S. S., Duitrk, R. A., and Smith, M. L., 1973, Sequencing research and the industrial scheduling problem, Symposium on the Theory of Scheduling and Its Applications, 29-38.

Raman, N., Talbot, r. B., and Rachamadugu, R. V., 1986, Simultaneous scheduling of machines and matcrial handling devices in automated manufacturing, Proceedings Second ORSA/TIMS Conference on Flexible Manufacturing Systems, K. E. Stecke and R. Suri (cds), 455-465 (Amsterdarn: Eilsevier).

SHAw, M., 1986, A pattern-directed approach to FMS scheduling, Proceedings Second ORSA/TIMS Conference on Flexible Manufacturing Systems, K. E. Stecke and R. Suri (eds), 545-554 (Amsterdam: Elsevier).

SHAw, M., 1987, A distributed scheduling method for computer integrated manufacturing: the use of local area nctworks in cellular systems, International Journal of Production Research, 25, 9, 1285-1303.

SHAw, M., 1988, Knowledge-based scheduling in flexible manufacturing systems: an integration of pattern directed inference and heuristic search, International Journal of Production Research, 26, 5, 82l-844. 
Shaw, M.J. P., and Whinston, A. B., 1986, Applications of artificial intelligence to planning and scheduling in lexible manufacturing systems, in A. Kusiak (ed.), Flexible Manufacturing Systems: Methods and Studies, 223-242 (Amsterdam: North-Holland).

Shen, S., and Chang, Y. L., 1986, An Al approach to schedule generation in a flexible manufacturing system, Proceedings Second ORSA/TIMS Conference on Flexible Manufacturing Systems, K. E. Stecke and R. Suri (eds), 581-592 (Amistcrdam: Flsevier).

SMith, M. L., Ramesh, R., DUdek, R. A., and Bl.AiR, E.I., 1986, Characteristics of US flexible manufacturing systems-a survey, Proceedings Second ORSA/TIMS Conference on Flexible Manufaciuring Systems, K. E. Stecke and R. Suri (eds), 477-486 (Amsterdam: Elsevier).

SMITH, M. L., and SfidMAnN, A., 1983, Due-date selection procedures for job-shop simulation, Comput. and Industrial Engineering, 7, 3, $199-207$.

STECKF, K. F., and SOLBERG, J. J., 1981, Loading and control policies for a flexible manufacturing system, International Journal of Production Research, 19, 5 .
Subramanyam, S., and Askin, R. G., 1986, An expert systems approach to scheduling in flexible manufacturing systems, in A. Kusiak (ed.), Flexible Manufacturing Systems: Methods and Studies, 243-256 (Amsterdam: North-Holland).

TANG, C. S., and Denardo, E. V., 1988a, Models arising from a flexible manufacturing machine, Part I: minimization of the number of tool switches, Operations Research, 36, 5, September-October, 767-777.

TA.NG, C. S., and IDENARDO, E. V., 1988b, Models arising from a flexible manufacturing machinc, Part II: minimization of the number of switching instants, Operalions Research, 36, 5, September--October, $778-784$.

VepsalainfN, A. P. J., and MORTON, T. E., 1988, Improving local priority rules and global lead-time estimates; a simulation study, Journal of Manufacturing and $O M, 1$, $102-118$.

WANG, H., 1986, An experimental analysis of flexible manufacturing systems (FMS), in A. Kusiak (ed.), Flexible Manufacturing Systems: Methods and Studies, 243-256 (Amsterdam: North-Holland). 